\title{
Defect models in silver halides
}

\author{
Y V G S MURTI* and RADHA D BANHATTI \\ Department of Physics, Indian Institute of Technology, Madras 600036, India \\ *Present address: Physics Department, Indian Institute of Technology, Guwahati 781001 , \\ India
}

\begin{abstract}
Silver halide crystals $\mathrm{AgCl}$ and $\mathrm{AgBr}$ exhibit all the three types of intrinsic point defects, namely, cationic Frenkel disorder, Schottky disorder and the bound vacancy pair disorder. The ionic conductivity anomaly at high temperatures, the unusual behaviour in respect of self- and impurity diffusion and the contrasting features in the anion self-diffusion between $\mathrm{AgCl}$ and $\mathrm{AgBr}$ do not all satisfactorily fit into the prevailing models of ionic transport. We review these features critically. We also discuss the potential and polarization models used in the theoretical evaluation of the enthalpies of formation of the intrinsic defects in these crystals. Results of our recent investigations on these questions will be presented to highlight their implications to the defect physics of these materials.
\end{abstract}

Keywords. Point defects; silver halides; formation enthalpies; diffusion; ionic conductivity.

\section{Introduction}

The quantitative role of point defects in mass and charge transport processes in ionic crystals is influenced by thermodynamic parameters such as the enthalpy and entropy of formation, migration and interaction of the defects. The defect concentrations follow an Arrhenius law with an activation energy governed by the enthalpy of formation of the intrinsic defects. Ionic conductivity studies on controlled crystal samples can be used to deduce these enthalpies after elaborate fitting of the data to a transport model via nonlinear regression analyses. Such an effort in favourable cases may yield reliable values of the thermodynamic parameters for the dominant defect disorder but information on the minority disorders cannot be deduced by such methods readily. Neutral defect species such as the bound vacancy pair only make a contribution to the matter transport and not to charge transport and are hence even more difficult to quantify experimentally. As a result, theoretical techniques of calculating the defect parameters come to the aid of the physicist in building complete models of ionic transport. It is well known that pure ab initio quantum mechanical methods are not adaptable to treating the defect problems in ionic crystals in view of the long range character of the defect field. One has therefore to take recourse to semiempirical techniques.

When one is to evaluate the enthalpy of any defect configuration, such as is associated with an isolated vacancy defect, the interstitial ion, or the saddle point configuration or the interaction between two point defects, the main issue to be settled is the relaxed configuration of the defect. The solving of this problem in an ionic crystal is really a subtle balancing act. Besides Coulomb interactions amongst all the point charges, there are many many-body forces which are at work: the overlapping charge distributions give rise to intermediate or long range forces of repulsive and attractive nature and these are not always additive. Further, the charged nature of the defect introduces a long range

\footnotetext{
*Author for correspondence
} 
polarization of two types: the lattice distortion associated with the inhomogeneous displacements of the ion centres and the deformation of the charge distribution of the ions themselves. These complicate an exact treatment of the problem.

Alkali halide crystals of the rock salt structure initially served as ideal prototype systems for modelling the ionic transport processes as well as for theoretical modelling. These crystals sustain Schottky defects as the main lattice disorder with little or negligible role of defects of other kinds. It was thus much easier to extract accurate values for the defect parameters from analyses of the transport data. Also the success of the theoretical techniques developed on these systems owes as much to the relative simplicity of the cohesion forces present in these crystals as to their moderate dielectric response. $\mathrm{AgCl}$ and $\mathrm{AgBr}$ also have the rocksalt structure and share several similar features with alkali halides. However the same techniques used for the alkali halides have proved inadequate since these materials exhibit a rich and complex defect structure due to the coexistence of several types of thermodynamic lattice disorder. Any realistic extension of defect models to more complex materials then warrants a more careful approach in (i) correctly representing the interionic interactions and in (ii) dealing with the larger question of the lattice and ionic distortions. Our recent experience leads us to think that $\mathrm{AgCl}$ and $\mathrm{AgBr}(\mathrm{AgX}$, $\mathrm{X}=\mathrm{Cl}, \mathrm{Br}$ ) provide a better prototype system for testing the efficacy of the defect models in these two respects.

\section{Ionic transport processes in $\mathrm{AgCl}$ and $\mathrm{AgBr}-\mathrm{A}$ review}

Experiments performed during the period 1930-1970 were directed towards resolving the question of the nature of the dominant species of lattice defects in AgX. The initial studies on the thermal expansion of the lattice parameter by Berry (1953) pointed to the existence of Schottky defects at the higher temperatures. The high temperature specific heat anomaly was attributed to cationic Frenkel defects (Kanzaki 1951). It was the pioneering work of Teltow and coworkers (Ebert and Teltow 1955) on the conductivity of $\mathrm{AgBr}$ doped with cadmium impurity ions that beautifully illustrated the law of mass action effect, with the silver interstitial ion emerging as the charge carrier more mobile than the vacancy.

Seitz (1951), in his celebrated review, advanced entropy arguments and used optical absorption spectra to argue in favour of the presence of Schottky defects in AgX at high temperatures. The experiments of Layer et al (1962) on the annealing of excess quenched-in conductivity of $\mathrm{AgX}$ have indeed provided strong indications for the existence of Schottky disorder. From their measurements of macroscopic and microscopic thermal expansion, Fouchaux and Simmons (1964) deduced limits for the concentration of Schottky vacancies vis-a-vis Frenkel defects. Near the melting temperatures, they deduce a Schottky defect density $x_{\mathrm{s}}<9 \times 10^{-5}$ whereas the density of the dominant cationic defects, $x_{F} \sim 10^{-3}$, placing the minority Schottky component to within an order of magnitude of the majority defect species. The investigations on the ionic conductivity in $\mathrm{AgX}$ thereafter took into account only the cationic Frenkel defects as the prevailing defect species. In treating the conductivity data to obtain defect parameters, one had to allow for the contributions of the two carriers viz. the cation vacancy and interstitial. The latter has multiple choices as possible jump paths: (i) the direct interstitial mechanism, (ii) the collinear and (iii) noncollinear interstitialcy 
mechanisms. The question of the relative importance of these paths however has not yet been unambiguously resolved (Slifkin 1991).

The biggest challenge for this model was the occurrence of anomalous excess conductivity near the melting point. Without abandoning the concept of a single lattice disorder on the cation sublattice, a mean field hypothesis (Aboagye and Friauf 1975), was introduced leading to a temperature dependent Gibbs free enthalpy of formation $g_{F}(T)$. This model has subsequently met with a number of hurdles when applied to the results on the diffusion of monovalent impurity ions in AgX (Laskar 1989). This class of impurity ions occupy substitutional sites in the lattice and their diffusion study provides a good probe on the cation vacancy defect. The diffusion of $\mathrm{Na}^{+}$and $\mathrm{K}^{+}$ions in $\mathrm{AgCl}$ did exhibit the expected high temperature curvature in the Arrhenius plot but in the case of $\mathrm{AgBr}$ the magnitude of the anomaly is much less than what is expected from the conductivity anomaly under the assumption of $g_{\mathrm{F}}(T)$. Besides, all the other impurity solutes failed to show any anomaly in diffusion behaviour, thus bringing the validity of the hypothesis of the $g_{F}(T)$ model into sharp focus. While the diffusion of divalent transition metal ions in $\mathrm{AgX}$ shows peculiarities, the anion self-diffusion and impurity diffusion on the anion sublattice present the most surprising facts. Ions of widely varying sizes do all have nearly the same activation energy! It cannot be denied that the diffusion of anions although comparatively slower than the diffusion of the silver ion, is a direct evidence for a significant degree of Schottky disorder. The Arrhenius plot for anion diffusion in $\mathrm{AgBr}$ shows curvature which was thought to be due to the contribution of vacancy pairs to the diffusion (Laskar 1989). The curvature is however absent in $\mathrm{AgCl}$. Further studies on doped crystals of $\mathrm{AgCl}$ have led to the conjecture that the pairs and single vacancies in $\mathrm{AgCl}$ have the same migration enthalpy (Batra and Slifkin 1969).

Based on an analysis of the conductivity data in $\mathrm{AgX}$, Andreoni and Tosi (1983) point out the fact that $\mathrm{AgX}$ exhibits a premelting behaviour rather than a transition to superionic behaviour. This is indicative of instability in both sublattices which can only follow from a Schottky type of disorder. If the difference in the formation enthalpies is of the order of $0.5 \mathrm{eV}$, and the entropy of Schottky defects is much larger than that of Frenkel defects, Schottky defects can well prevail at high temperatures leading to such an instability.

\section{The interionic potential in AgX}

A casual inspection of the many physical properties of $\mathrm{AgCl}$ and $\mathrm{AgBr}$ leaves one puzzled on the nature of the binding forces in these materials. Classified as 'essentially ionic', they have several features in common with the alkali halides. These include the crystal structure and optical transparency. Among the contrasting features are the large lattice energies, large dielectric constants and large Cauchy violations. Varied hypotheses had been advanced for these features: (i) the role of d-shell electrons and their covalency effects, (ii) quadrupolar deformability of the silver ion, (iii) many-body effects and (iv) strong van der Waals interactions. We have critically analyzed (Banhatti 1993) all these aspects from available evidence and have space only to report some of the main findings. Recent X-ray diffraction studies have led to accurate electron density maps which do not show accumulation of charge density along the bonds. This is supported by the charge densities deduced from the calculated 
electron band structures. Quadrupolar deformability of the silver ion was invoked (Kleppman 1976) to explain some properties of the phonon spectrum. Neither the physical basis for the dynamically deformed silver ion is supported nor the potential model based on this is free from unphysical features. The work of Bucher (1987) clearly brings to light the role of strong van der Waals forces as the cause of the contrasting characteristics of $\mathrm{AgX}$. By all accounts we have reasons to conclude that it is the unusually strong van der Waals interactions obtaining in $\mathrm{AgX}$ that are responsible for giving the semblance of covalency in these crystals which must be considered to be essentially ionic with a large van der Waals component of binding.

The most commonly used two-body central potential is of the form

$$
\phi_{i j}(r)=A_{i j} \exp \left[-\frac{r_{i j}}{\rho_{i j}}\right]-\frac{C_{i j}}{r_{i j}^{6}},
$$

the first representing the overlap repulsion due to Pauli exclusion and the second is the dipole-dipole type van der Waals (vdW) term. Catlow et al (1979) used such a potential with $A_{i j}$ and $\rho_{y}$ given from an electron gas model. In order to get the van der Waals coefficients $C_{t y}$, they attempted a fit to crystal properties in a shell model scheme which turned out to be poor. They then arbitrarily enhanced the coefficient $C_{\mathrm{CC}}$ for the silver-silver pairs. Neither the lattice energy nor the elastic constants agreed satisfactorily with the experimental values in this scheme. Jacobs et al (1977) employed such a potential to obtain a value of $1.39 \mathrm{eV}$ for $h_{\mathrm{F}}$ but the Schottky defect enthalpy turned out to be too large $(3 \cdot 0 \mathrm{eV})$.

Granzer et al (1980) supplemented the above form of the potential by a three-body vdW term following Axilrod and Teller and a three-body repulsion term following Sarkar and Sengupta. The parameters of the potential are obtained by fit to elastic constants, lattice energy, lattice parameter, and the transverse optic frequency. But the anion-anion repulsion strength parameter $A_{\mathrm{AA}}$ is found to be negative!. A more recent attempt by Baetzold et al (1989) incorporates angle-bending forces and triple dipole model. While they report satisfactory values of $h_{\mathrm{S}}$ with these three-body effects included, we note that surprisingly the Frenkel defect modelling did not need threebody forces at all.

We draw the following conclusions from the above review:

(I) There is a clear need to represent the van der Waals interactions fairly accurately in $\mathrm{AgX}$ if one were to simulate the defect environment satisfactorily.

(II) The fit to crystal properties tends to mask the real physical factors and introduces avoidable artifacts in the scheme. A minimal fit to crystal properties is desirable in constructing the potential.

\section{Our potentials for AgX}

Working in a simple effective two-body scheme of the form of (1) with the overlap potential parameters given by the Kim-Gordon (GK) type calculations, we have examined (Banhatti et al 1991) different ways of arriving at the dispersion parameters. In the potential of Set III of this reference, the three vdW coefficients were determined by using the lattice equilibrium condition at zero pressure and temperature together with the known mutual relations between $C_{i j}$ according to London's one-level formula. With the repulsion constans $A_{i j}$ and $\rho_{i j}$ obtained from electron gas type of calculations, 
we thus accomplish the task of obtaining all the important parameters of the potential with a minimal fit to crystal properties. We have found from this procedure that the van der Waals interactions are indeed strong in these crystals without any recourse to arbitrary hiking. We have also shown that this effective two-body potential can be reconciled with the failure of the Cauchy relations for the elastic constants, via the dynamic polarization model of Sangster (Banhatti 1993).

\section{Polarization model}

We have earlier remarked that the lattice and ion charge distributions are distorted on account of the inhomogeneous field around the charged defect. The polarization model is to be so chosen as to be consistent with the static dielectric response of the crystal in order to account for the contribution of these distorted charge distributions to the formation energy of the defect. In all earlier work, polarization was treated within the dipole approximation (Murti and Banhatti 1993).

In the extended point polarizable ion (EPPI) model we have recently introduced (Banhatti and Murti 1993), this has been refined to include the effects of the induced quadrupoles in the region 1 (nearest neighbours of the defect in our model) of a Mott-Littleton scheme of calculation. This is especially important for crystals with large dipole polarizabilities. There is an additional contribution $\left(W_{q}^{1}\right)$ to the formation enthalpy due to quadrupoles of region 1, which also affects the dipolar term through an extra field contribution.

$$
W_{\mathrm{q}}^{1}=-\frac{1}{2} \sum_{n}(1 / 6) \sum_{i j} Q_{i j} F_{m, i j},
$$

where the first summation is over the ions $(n)$ of region 1 and $F_{m, i j}$ the $i j$ component of the field gradient due to monopoles. The residual symmetry of the local defect environment has been fully exploited to simplify the problem. $Q_{i j}$ are the components of the quadrupole moment tensor. A complete account of the EPPI model and its application to the vacancy and interstitial defect are to be found in Banhatti and Murti (1993). The enthalpies of formation of the intrinsic disorders in $\mathrm{AgX}$ as evaluated using the MPPI and EPPI models are displayed in table 1. We see that one could hardly ignore the part of the polarization arising beyond the dipole approximation in these defect simulations.

\section{Conclusions}

A detailed examination of our results has shown (Banhatti 1993) that van der Waals forces have a key role to play in stabilizing the interstitial defect leading to the

Table 1.

\begin{tabular}{|c|c|c|c|c|c|c|}
\hline \multirow[b]{2}{*}{ Crystal } & \multicolumn{2}{|c|}{$h_{\mathrm{F}}(\mathrm{eV})$} & \multicolumn{2}{|c|}{$h_{\mathrm{s}}(\mathrm{eV})$} & \multicolumn{2}{|c|}{$h_{\mathrm{VP}}(\mathrm{eV})$} \\
\hline & MPPI & EPPI & MPPI & EPPI & MPPI & EPPI \\
\hline $\mathrm{AgCl}$ & $2 \cdot 42$ & 1.48 & $2 \cdot 73$ & 2.09 & 1.60 & $1 \cdot 1$ \\
\hline AgBr & $2 \cdot 34$ & $1 \cdot 34$ & $2 \cdot 47$ & 1.85 & $1 \cdot 26$ & 0.7 \\
\hline
\end{tabular}


dominance of Frenkel disorder. The values of $h_{\mathrm{S}}$ are only moderately larger than $h_{\mathrm{F}}$ (by $0.5-0.6 \mathrm{eV}$ ) and the values of $h_{\mathrm{Vp}}$ are quite small. It follows unmistakably that Schottky defects need to be taken seriously especially at the higher temperatures and vacancy pairs must exist in large numbers and play a more important role than previously envisaged in anion diffusion in $\mathrm{AgX}$. In the light of these findings a re-examination of the conductivity data in the anomaly region on one hand and a re-interpretation of the large body of diffusion data on the other is called for and it will help resolve the anomalies.

\section{References}

Aboagye J K and Friauf R J 1975 Phys. Rev. B11 1654

Andreoni W and Tosi M P 1983 Solid State Ionics 1149

Baetzold R C, Catlow C R A, Corish J, Healy F M, Jacobs P W M, Leslie M and Tan Y T 1989 J. Phys. Chem. Solids $\mathbf{5 0} 791$

Banhatti R D 1993 The role of induced quadrupoles in modelling intrinsic point defects of $\mathrm{AgCl}$ and $\mathrm{AgBr}$, Ph. D. thesis, IIT Madras

Banhatti R D and Murti Y V G S 1993 Phys. Rev. B48 6839

Banhatti R D, Murti Y V G S and Laskar A L 1991 Phys. Status Solidi b164 357

Batra A P and Slifkin L M 1969 J. Phys. Chem. Solids 301315

Berry C R 1953 Phys. Rev. 82422

Bucher M 1987 Phys. Rev. B35 6432

Catlow C R, Corish J and Jacobs P W M 1979 J. Phys. C12 3433

Ebert I and Teltow J 1955 Ann. Phys. 15258

Fouchaux R D and Simmons R O 1964 Phys. Rev. 1361664

Granzer F, Bucher M, Heuser H G, Petrasch P and Postada H M 1980 J. Phys. 41 C6-101

Jacobs P W M, Corish J and Catlow C R A 1980 J. Phys. C13 1977

Kanzaki H 1951 Phys. Rev. 81884

Kleppman W G 1976 J. Phys. C9 2285

Laskar A L 1989 in Superionic solids and solid electrolytes (eds) A L Laskar and S Chandra (London: Academic Press) p. 265

Layer H, Miller M G and Slifkin L M 1962 J. Appl. Phys. Suppl. 33478

Murti Y V G S and Banhatti R D 1993 Proc. int. conf. on defects in insulating materials (eds) $O$ Kanert and J-M Spaeth (Singapore: World Scientific) p. 198

Seitz F 1951 Rev. Mod Phys. 23328

Slifkin L M 1991 Philos. Mag. A64 1035 